\title{
Determining the aetiology of pulmonary oedema by the oedema fluid-to-plasma protein ratio
}

\author{
L.B. Ware*, R.D. Fremont*, J.A. Bastarache*, C.S. Calfee ${ }^{\#}$ and M.A. Matthay
}

ABSTRACT: We hypothesised that the oedema fluid-to-plasma protein (EF/PL) ratio, a noninvasive measure of alveolar capillary membrane permeability, can accurately determine the aetiology of acute pulmonary oedema.

390 mechanically ventilated patients with acute pulmonary oedema were enrolled. A clinical diagnosis of acute lung injury (ALI), cardiogenic pulmonary oedema or a mixed aetiology was based on expert medical record review at the end of hospitalisation. The EF/PL ratio was measured from pulmonary oedema fluid and plasma samples collected at intubation.

209 patients had a clinical diagnosis of $\mathrm{ALI}, 147$ had a diagnosis of cardiogenic pulmonary oedema and 34 had a mixed aetiology. The EF/PL ratio had an area under the receiver-operating curve of $\mathbf{0 . 8 4}$ for differentiating ALI from cardiogenic pulmonary oedema. Using a predefined cutoff of 0.65 , the EF/PL ratio had a sensitivity of $81 \%$ and a specificity of $81 \%$ for the diagnosis of ALI. An EF/PL ratio $\geqslant 0.65$ was also associated with significantly higher mortality and fewer ventilatorfree days.

Noninvasive measurement of the EF/PL ratio is a safe and reliable bedside method for rapidly determining the aetiology of acute pulmonary oedema that can be used at the bedside in both developed and developing countries.

KEYWORDS: Acute lung injury, acute pulmonary oedema, acute respiratory distress syndrome, alveolar capillary membrane permeability, diagnosis

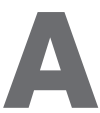
cute pulmonary oedema may be either due to increased permeability of the alveolar capillary barrier, in the case of acute lung injury (ALI) [1-3], or to increased pulmonary microvascular hydrostatic pressure [4], in the case of cardiogenic pulmonary oedema (CPE). Accurate determination of the aetiology of acute pulmonary oedema is of major clinical importance because the treatments for ALI and CPE are fundamentally different $[4,5]$. The shift in the practice of clinical medicine in both academic and nonacademic medical centres away from invasive measures, such as the pulmonary artery catheter, emphasises the need for other approaches to determine the clinical cause of pulmonary oedema.

Accurate and rapid determination of the cause of acute pulmonary oedema at the bedside can be challenging. Although history, physical examination and laboratory testing are useful, the aetiology of pulmonary oedema remains unclear in a significant number of patients even after initial diagnostic testing is completed [4], and clinical definitions are imperfect [6]. Echocardiography can provide information about left ventricular performance and filling pressures but is not rapidly available in many centres. The gold standard for determining the aetiology of acute pulmonary oedema is measurement of the pulmonary arterial occlusion pressure by pulmonary artery catheterisation [7, 8]. However, pulmonary artery catheterisation is invasive and has become much less common in the USA [9] with the publication of a number of studies suggesting that routine use of pulmonary artery catheterisation for the management of critically ill patients is associated with increased complications compared with central venous catheterisation and does not improve patient outcomes [1012]. Furthermore, a recent large multicentre trial of pulmonary arterial catheterisation found that close to one-third of patients with acute lung injury had elevated pulmonary arterial wedge pressures [12].

The pulmonary oedema fluid-to-plasma protein $(\mathrm{EF} / \mathrm{PL})$ ratio is a rapid, safe and noninvasive

\section{AFFILIATIONS}

${ }^{*}$ Division of Allergy, Pulmonary and Critical Care Medicine, Dept of Medicine, Vanderbilt University School of Medicine, Nashville, TN, and

${ }^{\#}$ Cardiovascular Research Institute and Depts of Medicine and Anesthesia, University of California, San Francisco, CA, USA.

CORRESPONDENCE

L.B. Ware

Division of Allergy, Pulmonary and Critical Care Medicine, Dept of Medicine

Vanderbilt University School of Medicine

T1218 MCN

116121 st Avenue S

Nashville

TN 37232-2650

USA

E-mail: lorraine.ware@vanderbilt.edu

Received:

June 232009

Accepted after revision:

Aug 272009

First published online:

Sept 092009 
measure of alveolar capillary membrane permeability [13]. The $\mathrm{EF} / \mathrm{PL}$ ratio can be measured inexpensively at the bedside and, thus, could be easily implemented as a diagnostic tool in both developing and developed countries. In addition, the EF/PL ratio can be measured at the onset of respiratory failure, immediately after intubation, providing critical diagnostic information long before other diagnostic test results are available. The EF/PL ratio was first proposed as a tool to determine the aetiology of acute pulmonary oedema in a study of 24 patients by FEIN et al. [14] in 1979. The EF/PL ratio has subsequently been used by our research group [15-18] and other investigators $[13,19-24]$ as a supplement to clinical data for determination of the aetiology of acute pulmonary oedema. Based on both clinical and experimental evidence, we have proposed that an EF/PL ratio $\geqslant 0.65$ is characteristic of patients with ALI whereas an EF/PL ratio $<0.65$ is characteristic of patients with CPE $[17,18,25]$. However, other than the original study by FEIN et al. [14], the clinical utility of the EF/PL ratio for determination of the cause of acute pulmonary oedema has never been validated. Validation in a larger group of patients is needed to assess the performance of this diagnostic test in a larger, more heterogeneous group of critically ill patients.

The primary goal of this study was to test the hypothesis that the EF/PL ratio, a noninvasive measure of the degree of alveolar capillary membrane permeability, can reliably differentiate the aetiology of acute pulmonary oedema in critically ill patients. To further assess the clinical utility of measuring the $\mathrm{EF} / \mathrm{PL}$ ratio, we also tested the association between the EF/PL ratio and important clinical outcomes, including mortality.

\section{METHODS}

\section{Patients}

Institutional review boards at the University of California San Francisco (UCSF; San Francisco, CA, USA) and Vanderbilt University (Nashville, TN, USA) approved the study with a waiver of informed consent. We studied 390 consecutively enrolled patients who were intubated and ventilated with positive pressure. These patients were included in a pulmonary oedema fluid databank at UCSF Moffitt-Long Hospital, San Francisco General Hospital and Vanderbilt University Medical Centre between 1981 and 2007. Criteria for enrolment in the databank included the acute onset of pulmonary oedema and mechanical ventilation. All patients in the database who had simultaneous samples of pulmonary oedema fluid and plasma were included in the current study. Some patients were included in prior reports [16-18, 25-27].

\section{Clinical diagnosis of the cause of pulmonary oedema}

The clinical aetiology of acute pulmonary oedema was determined by expert review of the medical record by the authors at the end of the hospitalisation and was based on clinical data available at discharge including history, physical findings, laboratory testing, findings on chest radiograph, fluid balance, echocardiography, other tests of cardiac function, pulmonary artery catheterisation, culture results, response to therapy, autopsy findings and impression of the treating physician. The clinical diagnosis was determined to be ALI when the standard American-European Consensus Definition [28] of ALI or acute respiratory distress syndrome was met in the setting of clinical findings consistent with sepsis, pneumonia, aspiration of gastric contents, severe trauma, multiple transfusions, reperfusion injury after lung transplantation, drug overdose, drug reaction or acute pancreatitis. The clinical diagnosis was determined to be CPE when the clinical findings were consistent with acute systolic or diastolic heart failure, acute myocardial infarction or acute volume overload. In a small subgroup of patients, the clinical findings were consistent with both ALI and CPE; this group was given a diagnosis of a mixed aetiology of pulmonary oedema. The expert reviewers were blinded to the EF/PL ratio.

\section{Sample collection for the EF/PL ratio}

Undiluted pulmonary oedema fluid and simultaneous plasma samples were obtained by previously described methods [15]. In brief, pulmonary oedema fluid was obtained by inserting a standard bedside suction catheter (usually 14 gauge) into the endotracheal tube and advancing the catheter into the distal airways. Gentle suction was then applied to remove 0.5$2.0 \mathrm{~mL}$ of free-flowing pulmonary oedema fluid that was suctioned directly into a standard suction trap. Next, a 3-mL blood sample was collected into a heparinised or EDTAtreated collection tube, usually from an existing arterial line or central venous line. Samples were centrifuged $(3,000 \times g$ for $10 \mathrm{~min})$, and the supernatant was stored at $-70^{\circ} \mathrm{C}$. The total protein was measured in both the oedema fluid and plasma by the Biuret method [15], and the EF/PL ratio was calculated as the oedema fluid protein concentration divided by the plasma protein concentration. The time from endotracheal intubation to aspiration of oedema fluid was recorded.

\section{Statistical analysis}

Statistical analysis was performed using statistical software (Stata/SE 9.2, College Station, TX, USA) and mean \pm SD or median with interquartile range (IQR) are reported as appropriate. ANOVA with the post hoc Tukey test was used to compare normally distributed continuous variables. For continuous variables that were not normally distributed, the Kruskall-Wallis test was used with a post hoc Bonferroni correction. Chi-squared analysis was used to compare categorical variables. Receiver operator curves (ROC) were generated using the EF/PL ratio as the predictor and the expert clinical diagnosis as the outcome. Cox proportional hazards regression was used to evaluate the impact of the EF/PL ratio on the per cent of patients alive and free of mechanical ventilation over the first 28 days. A p-value $<0.05$ was considered statistically significant.

\section{RESULTS}

\section{Patients}

390 patients with simultaneous samples of pulmonary oedema fluid and plasma were entered into the database over the study period. Baseline characteristics and clinical outcomes of the patients are listed in tables 1 and 2. Among the 209 patients with a clinical diagnosis of ALI, the most common underlying aetiology was pneumonia (28\%), followed by nonpulmonary sepsis $(26 \%)$, aspiration of gastric contents $(17 \%)$, drug reaction or overdose $(6 \%)$, multiple transfusions $(6 \%)$ or other causes $(17 \%)$, including reperfusion injury after lung transplantation, acute pancreatitis and severe trauma. Among the 147 patients with a clinical diagnosis of CPE, the most common underlying aetiology was acute myocardial infarction/ischaemia (33\%), 
followed by volume overload/diastolic dysfunction $(27 \%)$, congestive heart failure (16\%) and other causes (24\%), including valvular disease, arrhythmia, post-obstructive pulmonary oedema and neurogenic pulmonary oedema. The median (IQR) time from intensive care unit admission to endotracheal intubation was $0.0(0.0-4.25) \mathrm{h}$ in the group with a clinical diagnosis of CPE and $0.0(0.0-11.0) \mathrm{h}$ in the group with a clinical diagnosis of ALI. There were 34 patients in whom the clinical diagnosis determined at the end of the hospitalisation was consistent with a mixed aetiology of pulmonary oedema. These patients were excluded from the initial analysis of the diagnostic performance of the EF/PL ratio to discriminate between ALI and CPE.

\section{Comparison of EF/PL ratio to clinical diagnosis of $A L I$ versus $\mathrm{CPE}$}

The diagnostic performance of the EF/PL ratio was compared with the clinical diagnosis determined at the end of the hospitalisation using ROC analysis. Compared with the clinical diagnosis based on all information available at the end of the hospitalisation, the EF/PL ratio determined early in the hospital course had excellent diagnostic discrimination with an area under the curve (AUC) of 0.84 (95\% CI 0.79-0.88) (fig. 1a). In contrast, neither the oedema fluid protein level alone (AUC $0.73,95 \%$ CI $0.67-0.78$ ) or the plasma protein level alone (AUC 0.33, 95\% CI 0.27-0.39) provided the same degree of diagnostic discrimination. Because the oedema fluid protein level can rise over time if alveolar fluid clearance mechanisms are intact $[15,17]$, we repeated the analysis restricting inclusion to the 183 patients who had pulmonary oedema fluid sampled within $3 \mathrm{~h}$ of endotracheal intubation. In this group, the

\begin{tabular}{|c|c|c|c|}
\hline \multirow[t]{3}{*}{ TABLE 1} & $\begin{array}{l}\text { haracteristic } \\
\text { nonary oede } \\
\text { based on ex } \\
\text { he end of th }\end{array}$ & $\begin{array}{l}390 \text { patie } \\
\text { classified } \\
\text { review of } \\
\text { ospitalisat }\end{array}$ & $\begin{array}{l}\text { with } \\
\text { clinical } \\
\text { medical }\end{array}$ \\
\hline & \multicolumn{3}{|c|}{ Clinical diagnosis } \\
\hline & ALI & CPE & $\begin{array}{c}\text { Mixed } \\
\text { aetiology }\end{array}$ \\
\hline Subjects $n$ & 209 & 147 & 34 \\
\hline Age yrs & $47 \pm 18^{\star \star \star}$ & $55 \pm 20$ & $50 \pm 19$ \\
\hline Male & 58 & 54 & 53 \\
\hline Caucasian & 67 & 67 & 50 \\
\hline Nonsmoker & 73 & 73 & 71 \\
\hline LIS & $3.0 \pm 0.7^{\star \star \star}$ & $2.6 \pm 0.7$ & $2.7 \pm 0.7$ \\
\hline SAPS II & $52 \pm 20^{\star \star \star}$ & $43 \pm 14$ & $49 \pm 16$ \\
\hline $\begin{array}{l}\text { A-a oxygen difference } \\
\text { mmHg }\end{array}$ & $511 \pm 128$ & $487 \pm 135$ & $510 \pm 126$ \\
\hline EF/PL protein ratio & $0.89 \pm 0.36^{\# \# \#}$ & $0.53 \pm 0.21$ & $0.62 \pm 0.19$ \\
\hline \multicolumn{4}{|c|}{$\begin{array}{l}\text { Data are presented as mean } \pm \text { SD or } \% \text {, unless stated otherwise. ALI: acute lung } \\
\text { injury; CPE: cardiogenic pulmonary oedema; LIS: lung injury score; SAPS II: } \\
\text { simplified acute physiology score II; A-a oxygen difference: highest alveolar- } \\
\text { arterial oxygen difference on the day of oedema fluid sampling; EF/PL: oedema } \\
\text { fluid-to-plasma protein ratio. }{ }^{\star \star \star}: \mathrm{p}<0.001 \text { versus CPE group; }{ }^{\# \# \#: ~} \mathrm{p}<0.001 \\
\text { versus CPE group and mixed aetiology group. }\end{array}$} \\
\hline
\end{tabular}

diagnostic performance of the EF/PL ratio was similar with an AUC of 0.85 (95\% CI 0.79-0.91) (fig. 1b).

As a sensitivity analysis, the ROC analysis was repeated including the patients who had been classified as having a mixed aetiology of pulmonary oedema. Because all patients in this mixed aetiology group had clinical evidence of lung injury as well as hydrostatic causes of pulmonary oedema, the mixed oedema patients were classified as ALI for this analysis. In the entire patient cohort $(n=390)$, the EF/PL ratio determined early in the hospital course continued to perform well as a diagnostic test with an AUC of 0.81 (95\% CI 0.77-0.86) (fig. 1c). This analysis was also repeated with the sample restricted to those patients who had oedema fluid sampled within $3 \mathrm{~h}$ of endotracheal intubation. In this group $(n=200)$, the AUC was 0.81 (95\% CI 0.74-0.87) (fig. 1d).

Using a predefined cut-off of 0.65 , the sensitivity and specificity of the EF/PL ratio for diagnosing ALI among patients with acute pulmonary oedema was evaluated first with exclusion of the patients with a mixed aetiology of pulmonary oedema. The sensitivity of the cut-off of EF/ $P L \geqslant 0.65$ was $81 \%$ and the specificity was $81 \%$. When only patients with EF sampled $<3 \mathrm{~h}$ after endotracheal intubation were included, the sensitivity was $85 \%$ and the specificity was $83 \%$. We then repeated these analyses including the patients with a clinical diagnosis of a mixed aetiology of acute pulmonary oedema. In the entire cohort, the sensitivity was $75 \%$ and the specificity was $81 \%$. Restricting the analysis to patients with EF sampled within $3 \mathrm{~h}$ of intubation, the sensitivity was $75 \%$ and the specificity was $83 \%$.

\section{Association of EF/PL ratio with clinical outcomes}

To test the clinical relevance of using the EF/PL ratio to classify the aetiology of pulmonary oedema, we compared outcomes in patients with EF/PL above and below the cut-off of 0.65 . Major outcomes in the two groups are shown in table 3 and figure 2. Patients with $E F / P L \geqslant 0.65$ had worse clinical

\begin{tabular}{|c|c|c|c|c|}
\hline \multirow[t]{3}{*}{ TABLE 2} & \multicolumn{4}{|c|}{$\begin{array}{l}\text { Clinical outcomes of } 390 \text { patients with acute } \\
\text { pulmonary oedema classified by clinical } \\
\text { diagnosis based on expert review of the medical } \\
\text { record at the end of the hospitalisation }\end{array}$} \\
\hline & & \multicolumn{3}{|c|}{ Clinical diagnosis } \\
\hline & & ALI & CPE & $\begin{array}{c}\text { Mixed } \\
\text { aetiology }\end{array}$ \\
\hline \multicolumn{2}{|l|}{ Subjects $\mathbf{n}$} & 209 & 147 & 34 \\
\hline \multicolumn{2}{|c|}{ Ventilator-free days } & $0(0-17)^{\star \star \star}$ & $22(0-26)$ & $11(0-23)$ \\
\hline \multicolumn{2}{|c|}{ ICU-free days } & $0(0-16)^{\star \star \star}$ & $17(0-24)$ & $2(0-21)$ \\
\hline \multicolumn{2}{|c|}{ Hospital mortality } & $59 * \star \star$ & 32 & 47 \\
\hline
\end{tabular}

Data are presented as median (interquartile range) or \%, unless otherwise stated. ALI: acute lung injury; CPE: cardiogenic pulmonary oedema; ICU: intensive care unit. Comparison of ALI, CPE and mixed aetiology groups by Chisquared for hospital mortality. Ventilator-free days and ICU free days were compared across groups using the Kruskal-Wallis test. ***: $p<0.001$ versus CPE group. 

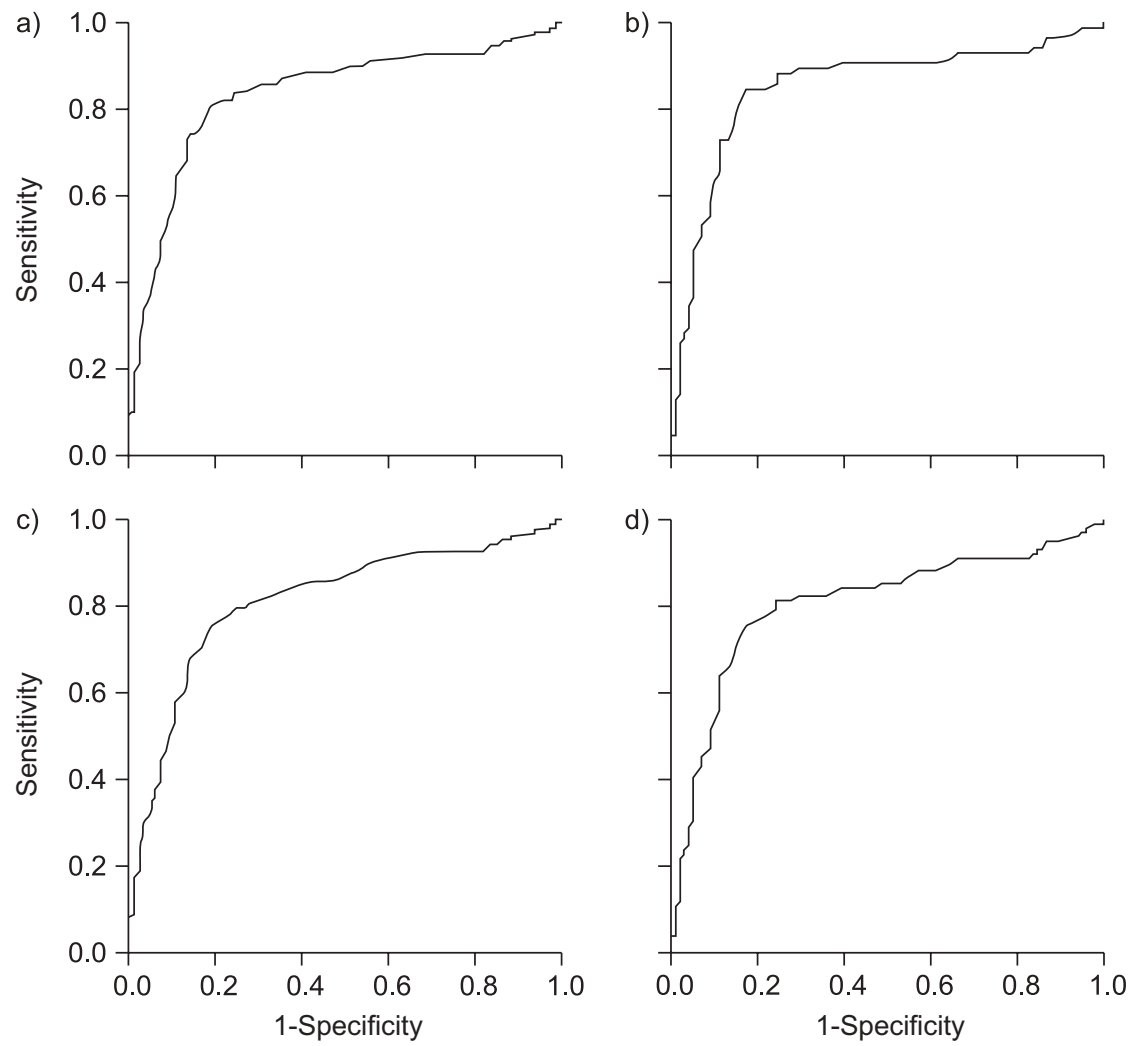

FIGURE 1. Receiver operator curve $(\mathrm{ROC})$ analyses of the utility of the oedema fluid-to-plasma protein (EF/PL) ratio for differentiating the aetiology of acute pulmonary oedema. a) Comparison of the EF/PL ratio to the clinical diagnosis based on all information available at the end of the hospitalisation, excluding patients with a clinical diagnosis of a mixed aetiology of acute pulmonary oedema $(n=356)$. The EF/PL ratio determined early in the hospital course had excellent diagnostic discrimination for acute lung injury (ALI) versus cardiogenic pulmonary oedema with an area under the curve (AUC) of $0.84(95 \% \mathrm{Cl} 0.79-0.88)$. b) The same analysis was restricted to the subgroup of patients who had pulmonary oedema fluid sampled within $3 \mathrm{~h}$ of endotracheal intubation $(n=183)$. In this group, the diagnostic performance of the $E F / P L$ ratio was similar with an AUC of 0.85 (95\% Cl 0.79-0.91). c) and d) For these analyses, the patients with a clinical diagnosis of a mixed aetiology of acute pulmonary oedema were classified as having ALI, and $\mathrm{c}$ ) the entire cohort $(\mathrm{n}=390)$ and $\mathrm{d}$ ) the subgroup with pulmonary oedema fluid sample within $3 \mathrm{~h}$ of endotracheal intubation ( $\mathrm{n}=200)$ were analysed. For the entire cohort, the AUC was 0.81 (95\% $\mathrm{Cl} 0.77-0.86$ ). For the subgroup with oedema fluid sampled within $3 \mathrm{~h}$ of endotracheal intubation the AUC was 0.81 (95\% $\mathrm{Cl} 0.74-0.87$ )

outcomes including mortality and number of ventilator-free days, particularly when the analysis was limited to patients in whom oedema fluid was sampled $<3 \mathrm{~h}$ after intubation (table 3).

\section{DISCUSSION}

The EF/PL ratio, a noninvasive measure of the degree of alveolar capillary membrane permeability, was first proposed as a clinical tool to differentiate the aetiology of acute pulmonary oedema by FeIN et al. [14] in 1979. In that study, oedema fluid and plasma protein concentrations were measured in 24 patients with acute pulmonary oedema. In the 20 patients with a clinical diagnosis of ALI, the EF/PL ratio was $\geqslant 0.65$ in all but three patients. In contrast, in the four patients with $\mathrm{CPE}$, the EF/PL ratio was $<0.65$ in all patients. Although the protein level in oedema fluid has subsequently been

\section{TABLE 3 Clinical outcomes of patients with acute pulmonary oedema classified by oedema fluid-to-plasma protein (EF/PL) ratio}

$\mathrm{EF} / \mathrm{PL}<0.65$

\section{All subjects}

Ventilator-free days

Hospital mortality \%

Subgroup with interval from intubation to oedema fluid sampling $<3 \mathrm{~h}$

Ventilator-free days

Hospital mortality \%

$\begin{array}{ccc}175 & 215 & \\ 10(0-26) & 0(0-22) & 0.006 \\ 50 & 61 & 0.022 \\ 103 & 97 & \\ 22(0-26) & 4(0-23) & <0.001 \\ 41 & 65 & 0.012\end{array}$

Data are presented as $n$ or median (interquartile range), unless otherwise stated. ${ }^{\#}$ : comparison of EF/PL groups by Chi-squared for hospital mortality. Ventilator-free days were compared using the Mann-Whitney U-test. 

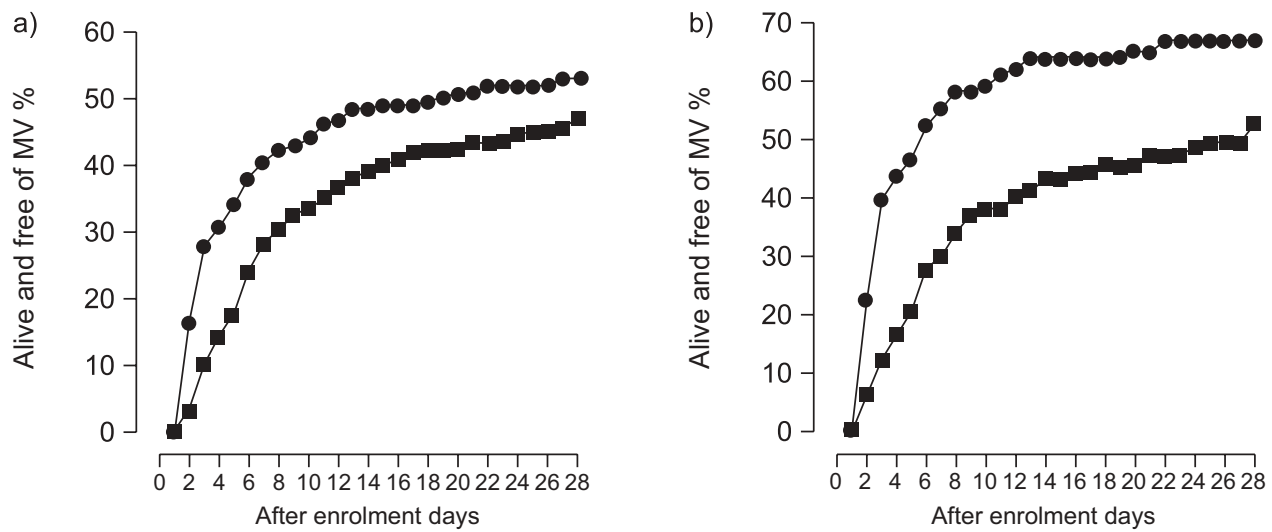

FIGURE 2. The percentage of patients alive and breathing without assistance during the first 28 days after enrolment. a) The entire cohort ( $n=389$ ), mortality data was missing for one subject. $p=0.058$ for oedema fluid-to-plasma protein $(E F / P L)$ ratio $<0.65$ ( $n=174)$ compared with $E F / P L \geqslant 0.65$ ( $n=215)$ by Cox proportional hazards regression analysis. b) Restricted to the subgroup $(n=200)$ in whom the oedema fluid was sampled within $3 \mathrm{~h}$ of endotracheal intubation. $p=0.005$ for $E F / P L<0.65$ ( $n=103$ ) compared with EF/PL $\geqslant 0.65(n=97)$ by Cox proportional hazards regression analysis. MV: mechanical ventilation. $\bullet: E F / P L<0.65 ; \mathbf{\square}: E F / P L \geqslant 0.65$.

measured experimentally in animal models of both ALI and $\mathrm{CPE}$ and has been used to differentiate the aetiology of acute pulmonary oedema in a number of clinical studies, it has never been validated in a large group of patients. Validation in a larger group of patients is needed to assess the performance of this diagnostic test in a larger more heterogeneous group of critically ill patients. In the current study of 390 critically ill patients, the EF/PL ratio had excellent diagnostic discrimination between ALI and CPE and was strongly associated with different clinical outcomes, confirming that the diagnostic classification of ALI versus CPE was clinically meaningful. These findings confirm the clinical relevance of the measurement of the EF/PL ratio in a large, heterogeneous group of patients with acute pulmonary oedema.

Differentiation of the aetiology of acute pulmonary oedema is of major clinical importance since the therapeutic approaches to the two most common aetiologies of acute pulmonary oedema, CPE and ALI are fundamentally different [4]. Patients with CPE require therapy to optimise cardiac function that includes therapies targeted at reduction of cardiac preload and/or afterload and optimisation of myocardial performance [29]. The underlying cause of CPE (myocardial infarction, congestive heart failure, diastolic dysfunction, acute volume overload) may have additional implications for treatment. Treatment of ALI should focus first and foremost on the search for underlying cause [5]. A source of infection should be sought in all patients since pulmonary and nonpulmonary sepsis are by far the most common causes of ALI. Lung protective ventilation is life saving and should be instituted promptly in all patients with ALI [30]. In addition, patients who have severe sepsis as the underlying cause of lung injury may be candidates for treatment with recombinant activated protein C (drotrecogin-alfa activated) [31]. In the absence of shock and tissue hypoperfusion, patients with ALI should be treated with a conservative fluid strategy [32].

Pulmonary oedema fluid is an underutilised diagnostic specimen that is readily and safely available and requires no special equipment for collection. Although the plasma and oedema fluid concentrations of protein in the current study were determined using a laboratory assay, the concentrations of protein can also be measured at the bedside after centrifugation using a hand-held refractometer. LIEN et al. [13] reported excellent correlation $(\mathrm{r}=0.991)$ between protein concentrations measured by refractometry and standard laboratory assay in pulmonary oedema fluid and plasma. As a safe, noninvasive, bedside test for the aetiology of pulmonary oedema, widespread use of the EF/PL would be tremendously beneficial in facilitating rapid institution of appropriate therapy directed at the underlying cause of acute pulmonary oedema, particularly in developing countries where other diagnostic tools such as pulmonary artery catheterisation and echocardiography may have limited availability. The ease with which the EF/PL ratio can be measured compares favourably with the comparison of pleural fluid and plasma protein concentrations, one of the primary tools for diagnostic classification of the aetiology of pleural effusion [33]. Of note, pleural fluid protein concentrations have also been measured at the bedside using refractometry [34].

The primary limitation of this study is the retrospective nature of the review of the medical record for the clinical classification of the aetiology of pulmonary oedema, although the study analysis plan was designed prospectively before medical charts were reviewed. Medical records were reviewed at the end of the hospitalisation to allow all available clinical information, including autopsy findings, to be considered in the clinical diagnosis. To avoid being biased by the EF/PL ratio, the investigators were not aware of the EF/PL ratios while carrying out the chart review. A second limitation is that pulmonary oedema fluid was not sampled immediately after endotracheal intubation in every patient. The median time between endotracheal intubation and sampling of pulmonary oedema fluid was $2.5 \mathrm{~h}$. Delays in collection of oedema fluid can lead to elevated EF/PL ratios in patients who have intact alveolar fluid clearance mechanisms. Over time, water and solute is absorbed faster than protein, thereby concentrating the protein within the alveolar space [35]. Thus, delays in sampling of pulmonary oedema fluid could lead to misclassification of patients. For example, a patient with pure 
hydrostatic pulmonary oedema could be classified erroneously as a mixed aetiology. This is the likely explanation for the slightly better diagnostic performance of the EF/PL ratio when the analysis was restricted to the group of patients with oedema fluid sampled within $3 \mathrm{~h}$ of endotracheal intubation. A third potential limitation is that patients were not protocolised to different diagnostic strategies in a way that would assess the relative value of the EF/PL ratio compared with other diagnostic modalities. Although some prior reports have assessed the contribution of individual diagnostic tests such as the chest radiograph [36] or plasma N-terminal brain naturitic protein (BNP) [37], no prior studies have systematically assessed the specific contribution of all the available diagnostic tests in a time-dependent analysis in patients with acute pulmonary oedema. We believe that the current study has validity and practical value because the results demonstrate the value of a noninvasive test that can be performed rapidly, similar to the widely accepted diagnostic classification of pleural effusions as transudates or exudates based on pleural fluid and plasma protein concentrations, a classification that is used to guide further diagnostic tests and therapy for pleural effusions.

Although exact estimates of the global incidence of acute pulmonary oedema are not available, data from the World Health Organization's Global Burden of Disease Study consistently place ischaemic heart disease, a common cause of $\mathrm{CPE}$, and lower respiratory infections, a common cause of ALI, in the top five causes of death worldwide both in the original study [38] and in projections to the year 2030 [39]. In this study and others, both ALI and acute CPE are associated with high rates of morbidity and mortality, highlighting the need for prompt recognition and institution of appropriate therapy. Our findings indicate that the EF/PL ratio can be used to determine the aetiology of acute pulmonary oedema. The rapid, safe, noninvasive nature of this diagnostic test renders it highly appealing for routine use in both developed and developing countries. Since the accepted treatment strategies for cardiogenic and noncardiogenic pulmonary oedema are different [4,5], rapid noninvasive determination of the cause of pulmonary oedema can direct the clinician to the correct therapies, an especially important objective in critically ill, ventilated patients with acute pulmonary oedema. Because the $\mathrm{EF} / \mathrm{PL}$ ratio can be measured at the onset of respiratory failure, immediately after intubation, it can provide this critical diagnostic information, long before other diagnostic test results are available. Future studies could test the contributions of the different diagnostic tests for distinguishing cardiogenic and noncardiogenic pulmonary oedema, including the ECG, the portable chest radiograph, the plasma levels of troponin and $\mathrm{BNP}$, and the EF/PL ratio in ventilated patients with acute pulmonary oedema.

\section{SUPPORT STATEMENT}

This work was supported by National Institute of Health (Bethesda, MD, USA) grants HL 081332, HL 51856 and HL090833 awarded to L.B. Ware, M.A. Matthay and C.S. Calfee, respectively. C.S. Calfee was supported by the Flight Attendant Medical Research Institute (Miami, FL, USA).

\section{STATEMENT OF INTEREST}

None declared.

\section{REFERENCES}

1 Ashbaugh DG, Bigelow DB, Petty TL, et al. Acute respiratory distress in adults. Lancet 1967; 2: 319-323.

2 Piantadosi CA, Schwartz DA. The acute respiratory distress syndrome. Ann Intern Med 2004; 141: 460-470.

3 Wyncoll DL, Evans TW. Acute respiratory distress syndrome. Lancet 1999; 354: 497-501.

4 Ware LB, Matthay MA. Clinical practice. Acute pulmonary edema. N Engl J Med 2005; 353: 2788-2796.

5 Ware LB, Matthay MA. Medical progress: the acute respiratory distress syndrome. N Engl J Med 2000; 342: 1334-1349.

6 Esteban A, Fernandez-Segoviano P, Frutos-Vivar F, et al. Comparison of clinical criteria for the acute respiratory distress syndrome with autopsy findings. Ann Intern Med 2004; 141: 440-445.

7 Swan HJC, Ganz W, Forrester J, et al. Catheterization of the heart in man with use of a flow directed balloon-tipped catheter. $N$ Engl J Med 1970; 283: 447-451.

8 Matthay MA, Chatterjee K. Bedside catheterization of the pulmonary artery: risks compared with benefits. Ann Intern Med 1988; 109: 826-834.

9 Wiener RS, Welch HG. Trends in the use of the pulmonary artery catheter in the United States, 1993-2004. JAMA 2007; 298: 423-429.

10 Binanay C, Califf RM, Hasselblad V, et al. Evaluation study of congestive heart failure and pulmonary artery catheterization effectiveness: the ESCAPE trial. JAMA 2005; 294: $1625-1633$.

11 Harvey S, Harrison DA, Singer M, et al. Assessment of the clinical effectiveness of pulmonary artery catheters in management of patients in intensive care (PAC-Man): a randomised controlled trial. Lancet 2005; 366: 472-477.

12 Wheeler AP, Bernard GR, Thompson BT, et al. Pulmonary-artery versus central venous catheter to guide treatment of acute lung injury. N Engl J Med 2006; 354: 2213-2224.

13 Lien TC, Wang JH. Rapid differentiation of pathogenesis in patients with pulmonary edema by protein measurement with refractometer. Chin Med J (Taipei) 1995; 56: 298-304.

14 Fein A, Grossman RF, Jones JG, et al. The value of edema protein measurements in patients with pulmonary edema. Am J Med 1979; 67: 32-39.

15 Matthay MA, Wiener-Kronish JP. Intact epithelial barrier function is critical for the resolution of alveolar edema in humans. Am Rev Respir Dis 1990; 142: 1250-1257.

16 Ware LB, Golden JA, Finkbeiner WE, et al. Alveolar epithelial fluid transport capacity in reperfusion lung injury after lung transplantation. Am J Respir Crit Care Med 1999; 159: 980-988.

17 Ware LB, Matthay MA. Alveolar fluid clearance is impaired in the majority of patients with acute lung injury and the acute respiratory distress syndrome. Am J Respir Crit Care Med 2001; 163: 1376-1383.

18 Verghese GM, Ware LB, Matthay BA, et al. Alveolar epithelial fluid transport and the resolution of clinically severe hydrostatic pulmonary edema. J Appl Physiol 1999; 87: 1301-1312.

19 Sprung C, Rackow E, Fein I, et al. The spectrum of pulmonary edema: differentiation of cardiogenic intermediate and noncardiogenic forms of pulmonary edema. Am Rev Respir Dis 1981; 124: 718-722.

20 Carlson RW, Schaeffer RC Jr, Carpio M, et al. Edema fluid and coagulation changes during fulminant pulmonary edema. Chest 1981; 79: 43-49.

21 Carlson RW, Schaeffer RC Jr, Michaels SG, et al. Pulmonary edema fluid. Spectrum of features in 37 patients. Circulation 1979; 60: 1161-1169.

22 Carlson RW, Schaeffer RC Jr, Michaels SG, et al. Pulmonary edema following intracranial hemorrhage. Chest 1979; 75: 731-734. 
23 Mathru M, Blakeman B, Dries DJ, et al. Permeability pulmonary edema following lung resection. Chest 1990; 98: 1216-1218.

24 Katz S, Aberman A, Frand UI, et al. Heroin pulmonary edema. Evidence for increased pulmonary capillary permeability. Am Rev Respir Dis 1972; 106: 472-474.

25 Smith WS, Matthay MA. Evidence for a hydrostatic mechanism in human neurogenic pulmonary edema. Chest 1997; 111: 1326-1333.

26 Fremont RD, Kallet RH, Matthay MA, et al. Postobstructive pulmonary edema: a case for hydrostatic mechanisms. Chest 2007; 131: 1742-1746.

27 Sue RD, Matthay MA, Ware LB. Hydrostatic mechanisms may contribute to the pathogenesis of human re-expansion pulmonary edema. Intensive Care Med 2004; 30: 1921-1926.

28 Bernard GR, Artigas A, Brigham KL, et al. The AmericanEuropean Consensus Conference on ARDS. Definitions, mechanisms, relevant outcomes, and clinical trial coordination. Am J Respir Crit Care Med 1994; 149: 818-824.

29 Mebazaa A, Gheorghiade M, Pina IL, et al. Practical recommendations for prehospital and early in-hospital management of patients presenting with acute heart failure syndromes. Crit Care Med 2008; 36: Suppl. 1, S129-S139.

30 The Acute Respiratory Distress Syndrome Network. Ventilation with lower tidal volumes as compared with traditional tidal volumes for acute lung injury and the acute respiratory distress syndrome. N Engl J Med 2000; 342: 1301-1308.
31 Bernard G, Vincent J-L, Laterre P-F, et al. Efficacy and safety of recombinant human activated protein $\mathrm{C}$ for severe sepsis. $\mathrm{N}$ Engl J Med 2001; 344: 699-709.

32 Wiedemann HP, Wheeler AP, Bernard GR, et al. Comparison of two fluid-management strategies in acute lung injury. $N$ Engl J Med 2006; 354: 2564-2575.

33 Porcel JM, Light RW. Diagnostic approach to pleural effusion in adults. Am Fam Physician 2006; 73: 1211-1220.

34 Light RW. Falsely high refractometric readings for the specific gravity of pleural fluid. Chest 1979; 76: 300-301.

35 Matthay MA, Folkesson HG, Clerici C. Lung epithelial fluid transport and the resolution of pulmonary edema. Physiol Rev 2002; 82: 569-600.

36 Aberle DR, Wiener-Kronish JP, Webb WR, et al. Hydrostatic versus increased permeability pulmonary edema: diagnosis based on radiographic criteria in critically ill patients. Radiology 1988; 168: 73-79.

37 Januzzi JL Jr, Camargo CA, Anwaruddin S, et al. The N-terminal Pro-BNP investigation of dyspnea in the emergency department (PRIDE) study. Am J Cardiol 2005; 95: 948-954.

38 Murray CJ, Lopez AD. Global mortality, disability, and the contribution of risk factors: Global Burden of Disease Study. Lancet 1997; 349: 1436-1442.

39 Mathers CD, Loncar D. Projections of global mortality and burden of disease from 2002 to 2030. PLoS Med 2006; 3: e442. 